Histoire de l'éducation

DEDTCATION

$126 \mid 2010$

Ecole, histoire et nation

\title{
Présentation
}

\section{Antoine Prost}

\section{(2) OpenEdition}

Journals

Édition électronique

URL : https://journals.openedition.org/histoire-education/2146

DOI : 10.4000/histoire-education.2146

ISSN : 2102-5452

\section{Éditeur}

ENS Éditions

\section{Édition imprimée}

Date de publication : 1 avril 2010

Pagination : $5-10$

ISSN : 0221-6280

\section{Référence électronique}

Antoine Prost, « Présentation », Histoire de l'éducation [En ligne], 126 | 2010, mis en ligne le 17

novembre 2010, consulté le 20 mai 2021. URL : http://journals.openedition.org/histoire-education/

2146 ; DOI : https://doi.org/10.4000/histoire-education.2146 


\section{Présentation}

\section{Antoine PROST}

Cette livraison d'Histoire de l'éducation est quelque peu inhabituelle. Elle s'inscrit en contrepoint des actes d'un séminaire organisé par l'INRP à Lyon, le $1^{\text {er }}$ avril dernier, sur "L'École et la Nation". Il nous a semblé intéressant de reprendre certaines des contributions à ce séminaire pour les approfondir, et de les compléter par des articles supplémentaires, tout en leur donnant une orientation un peu différente. C'est ce que résume le titre de ce numéro: "École, histoire et nation".

La problématique du séminaire était plus générale. Bien que l'histoire y tînt une grande place, elle n'était pas seule concernée : sa compagne de toujours en France, du moins - la géographie, l'accompagnait. Surtout, il partait du constat que l'école n'était pas le seul creuset de la nation, comme on le croit trop souvent. Beaucoup d'autres institutions ont contribué à créer la nation française : l'administration, la monnaie, les poids et mesures, le service militaire, etc. La nation existait avant l'école et d'une certaine façon, elle l'a créée à son image.

Le numéro qu'on va lire ne revient pas sur ces questions générales. Il s'attache au discours historique tenu par l'école. Mais cette focalisation disciplinaire est compensée par une extension chronologique : les articles ici réunis vont du XIX ${ }^{\mathrm{e}}$ siècle à 2010. Outre la France, ils concernent l'Espagne, le Canada, et bien au-delà dans l'article de Patrick Cabanel ${ }^{1}$. De leur confrontation se dégagent des conclusions attendues et d'autres qui le sont moins.

\section{Histoire et création du sentiment national}

Personne ne sera surpris du rôle considérable que les États examinés dans

"École et nation : l'exemple des livres de lecture scolaires (XIX et première moitié du XXe siècles)", infra, p. 33-54. 
ce volume accordent à l'histoire dans la création du sentiment national. Il y a là un effet de construction : on ne peut étudier l'enseignement de l'histoire que dans les pays où il existe, et s'il y existe, c'est précisément parce que ces pays lui accordent une efficacité sociale, civique et nationale. En ce sens, l'histoire enseignée à l'école l'est toujours pour autre chose que l'histoire ; elle est toujours peu ou prou instrumentalisée ; Lucien Febvre dirait qu'elle est une histoire " serve " ${ }^{2}$, qu'il s'agisse à travers elle de former des petits Français, Italiens, Suédois, Québécois et Catalans, ou plus généralement de futurs citoyens en développant leur esprit critique.

Pour banal qu'il soit, ce constat n'en pose pas moins une question : pourquoi des pays comme la Grande-Bretagne ou les États-Unis n'accordent-ils dans leurs curricula qu'une place marginale à l'histoire? Est-ce parce qu'ils font confiance à d'autres pédagogies pour former leurs nationaux ? En montrant le succès mondial de la formule du "tour de la nation ", Patrick Cabanel nous rappelle que l'apprentissage de la patrie passe également par la découverte de son territoire et, à travers lui, de ses activités, de sa culture, de sa diversité, et que ces lectures sont beaucoup plus un complément qu'une alternative à l'enseignement de l'histoire nationale. Ou peut-on dire que la nation n'a pas pour les Britanniques et les Américains la même importance que pour les Français, les Québécois, les Suédois ? Que leur conception de la nation est différente ? Poser ces questions oblige à s'interroger sur le concept même de nation : que met l'enseignement de l'histoire derrière ce mot, reçu sur le mode de l'évidence?

Cette interrogation est au cœur même de l'article d'Olivier Loubes ${ }^{3}$. En distinguant le corps physique de la nation - territoire, population, économie, gouvernement et institutions, etc. - et son corps sacré, les valeurs qui s'imposent à tous, il soulève une question centrale. Enseigner le corps physique de la nation est affaire de connaissance et d'intelligence. Mais cela ne suffit pas pour enseigner son corps sacré ; à dire vrai, il ne s'enseigne pas ; il doit devenir

2 Lucien Febvre, "L'histoire dans le monde en ruines ", Leçon d'ouverture du cours d'histoire moderne à l'Université de Strasbourg, Revue de synthèse historique, février 1920, p. 4: "L'histoire qui sert, c'est une histoire serve". 
l'objet d'un attachement affectif. Il faut le faire aimer. On pourrait reprendre ici la phrase célèbre de Lavisse : "L'amour de la patrie ne s'apprend pas par cœur ; il s'apprend par le cœur ", ou encore ces maximes : "à l'enseignement historique incombe le devoir de faire aimer et de faire comprendre la patrie. [...] N'enseignons point l'histoire avec le calme qui sied à l'enseignement des participes. Il s'agit ici de la chair de notre chair et du sang de notre sang " ${ }^{5}$.

Les républicains ont ainsi opéré un véritable transfert de sacralité, de l'histoire sainte qui s'enseignait jusqu'à la laïcisation de 1882, à l'histoire d'une patrie phare de l'humanité, car, comme le rappelle Annie Bruter ${ }^{6}$, ils n'ont inventé ni l'enseignement de l'histoire, ni ses finalités patriotiques. Ils ont substitué un patriotisme républicain à un patriotisme conservateur-catholique, dans les écoles publiques du moins, car dans l'enseignement catholique, ce dernier semble avoir résisté jusque dans l'entre-deux-guerres. Olivier Loubes, qui nous le fait remarquer, explique la profondeur de la mobilisation de 1914 par la conjonction de ces deux histoires patriotiques toutes deux, chacune à leur manière : celle de la France fille aînée de l'Église, et celle de la patrie des droits de l'homme, héritière de 1789 et phare de l'humanité. Il y a là deux lectures différentes d'une même histoire, qui donnent d'ailleurs deux significations contrastées à l'immense deuil d'après-guerre, comme en témoigne la statuaire des monuments aux morts, du poilu victorieux brandissant fièrement une couronne de lauriers, au poilu agonisant dans les plis d'un drapeau tandis qu'un ange, un saint ou le Christ lui-même, dans certains vitraux d'église, se penche sur lui pour l'accueillir.

Par l'horreur que suscitent les millions de morts, de blessés, de veuves et d'orphelins, la Grande Guerre provoque un nouveau transfert de sacralité. Si l'histoire continue à enseigner le corps physique de la nation, elle ne diffuse plus tout à fait le même message moral. C'est toujours la patrie des droits de l'homme, mais le sacré patriotique, déjà partiellement contesté avant guerre, est réinterprété dans un sens pacifiste et internationaliste : la France est mainte-

4 Ernest Lavisse, Inauguration de l'école de Nouvion-en-Thiérache, À propos de nos écoles, Paris, A. Colin, 1895, p. 14.

5 Questions d'enseignement national, Paris, A. Colin, 1885, p. 208 et p. 210.

6 "L'enseignement de l'histoire nationale à l'école primaire avant la III République ", infra, p. 11-31. 
nant la nation qui veut la paix comme fin et l'organisation internationale comme moyen. La nation ne se dissout pas dans la SDN, mais elle tend vers elle. Cette foi vient buter sur la montée des fascismes et la venue de la Seconde Guerre mondiale, qui l'empêchent de se consolider, mais derrière cette réinterprétation pacifiste, la culture républicaine subsiste, profondément enracinée.

\section{La désacralisation de l'État-nation}

On peut alors se demander si, les années noires passées, l'enseignement de l'histoire a retrouvé une France à faire aimer aux élèves. La question appellerait une recherche approfondie que personne n'a encore conduite, à ma connaissance. Aussi ne puis-je que proposer deux hypothèses apparemment contradictoires. La première est que la professionnalisation de l'histoire, amorcée dès le $\mathrm{XIX}^{\mathrm{e}}$ siècle, a conduit alors les enseignants à refuser cette instrumentalisation : c'est un rôle qu'ils n'ont plus voulu jouer. La seconde, est que la magnification de la Résistance, que certains aujourd'hui jugent abusive au point de tomber dans l'excès contraire, a constitué le nouveau visage du corps sacré de la nation, jusqu'au moment où ce corps sacré s'est progressivement évanoui.

Que nous montrent, en effet, les trois études les plus contemporaines de ce numéro ? Joan Pagès Blanch ${ }^{7}$ analyse le cas de l'Espagne, où l'enseignement de l'histoire n'est qu'un élément du conflit beaucoup plus large entre deux nationalismes : celui d'une Catalogne qui veut s'affirmer comme telle et celui d'un État central qui s'y oppose. L'objectif est la catalanité de l'école, et non seulement l'histoire : il s'agit bien davantage de la langue, de la formation, des méthodes, et avant tout des autorités compétentes. On retrouverait sans doute des processus analogues dans les États issus de la décolonisation.

Le cas du Québec est différent, car le fédéralisme canadien laisse aux différentes provinces la compétence en matière d'éducation. On est loin du centralisme madrilène. Les décisions ne se prennent pas à Ottawa mais à Montréal, ce qui rend moins vifs les conflits. L'enseignement de l'histoire y est dominé jusqu'au milieu des années 1890 par ce que Jocelyn Létourneau ${ }^{8}$ appelle un " récit

\footnotetext{
7 "L'école et la question nationale en Catalogne (XVIII $-\mathrm{XX}^{\mathrm{e}}$ siècle) ", infra, p. 77-95.

8 "Quelle histoire d’avenir pour le Québec ?", infra, p. 97-119.
} 
du Soi " qui vise à renforcer l'identité francophone et catholique d'une nation entravée dans son affirmation et son développement par une domination anglophone et protestante. Puis les transformations de la population et de la société, jointes aux évolutions politiques, font prévaloir, non sans débats, un changement assez radical. Il ne se contente pas en effet de laïciser le programme et de l'ouvrir sur la Fédération et sur le monde : il modifie jusqu'à l'intitulé de cet enseignement qui devient " Histoire et éducation à la citoyenneté ".

Le rapprochement s'impose avec le récit que donne Patricia Legris ${ }^{9}$ de la lente et réticente ouverture des programmes d'histoire en France sur l'Europe et sur les différentes composantes d'une population qui se diversifie et où des communautés multiples revendiquent leur reconnaissance. Il me semble, en effet, que dans les deux cas on assiste à une progressive désacralisation de l'État-Nation. L'amour sacré de la patrie qu'invoque notre hymne national érige l'État-Nation en personne morale entièrement souveraine, seule juge des intérêts de la communauté qu'elle régit, et capable d'exiger de ses membres leur sacrifice. "Pour elle un français doit mourir ", disait le Chant du départ. Or le droit a évolué, et la communauté internationale limite la souveraineté des nations, comme on le voit avec la Cour européenne des droits de l'Homme et la Cour de justice de l'Union européenne, qui peut donner raison à un citoyen contre son État. Les États-Nations européens se réduisent progressivement, ce qui était déjà la conception d'États fédérés comme la Suisse ou le Canada, à une fonction de gestionnaires de services publics. On demande à l'histoire d'apprendre aux enfants et aux jeunes à vivre ensemble dans le respect mutuel de leurs personnalités et de leurs identités multiples. C'est ce que désigne "l'éducation à la citoyenneté". Les préoccupations ont changé; le souci d'affirmation face aux autres nations par l'enseignement d'un grand récit national s'évanouit. Les objectifs internes l'emportent sur les fiertés internationales.

Il semble donc bien qu'il faille renverser les perspectives. On croit trop vite que l'enseignement de l'histoire fonde le sentiment national et soude la nation. À ce compte, l'État franquiste aurait dû réussir dans sa tentative pour réduire les régionalismes. Il n'y est pas parvenu, bien que son action ait duré beaucoup

9 "Les programmes d'histoire en France: la construction progressive d'une "citoyenneté plurielle" (1980-2010) ", infra, p. 121-151. 
plus - trente-six ans - que celle de Vichy en France. L'action volontariste de l'État doit son efficacité au consensus social qu'il est capable ou non de mobiliser. L'enseignement de l'histoire est un outil parmi d'autres que se donne une société pour atteindre ses objectifs, et les modalités de cet enseignement évoluent, avec un certain décalage chronologique, en fonction de l'évolution même des sociétés. Il est vain de chercher un rapport linéaire de l'État à l'enseignement de l'histoire puis à la nation et au sentiment national ou d'imaginer une causalité circulaire. Au vrai, cette problématique jacobine néglige la société civile. Déjà contestable avant la guerre de 1940, elle ne répond plus aux questions que pose aujourd'hui la mondialisation à des États qui ont perdu une partie de leur souveraineté.

Antoine PROST

Professeur émérite, université Paris 1-Panthéon-Sorbonne ap-prost@3dnet.fr 